\title{
FORT RODD HILL N.H.S.C.: WORKFLOW AND BENEFITS IN THE USE OF PHOTOGRAMMETRIC AND LIDAR DOCUMENTATION FOR THE PRODUCTION OF CONSTRUCTION TENDER DOCUMENTS
}

\author{
C.S.Kretz \\ Heritage Conservation Services, Public Services and Procurement Canada, 30 rue Victoria, K1A 0M6, \\ Gatineau, Canada - Shawn.Kretz@tpsgc-pwgsc.gc.ca \\ Commission II
}

KEY WORDS: Photogrammetry, Rehabilitation, 3D Modelling, Laser Scanning, UAV Documentation

\begin{abstract}
:
This case study examines the workflow, benefits, disadvantages and limitations of photogrammetry and laser scanning for the production of contract tender documents. This includes the use of the outputs of the aforementioned methodologies for direct inclusion in the construction tender drawing package. This study will also delve into evolving data capture and post production techniques, specifically the utilization of UAV's to further refine, supplement and provide greater efficiency in the field for the acquisition of photogrammetric data. Future phases of this project will leverage emerging technologies to further improve and expand upon the success of the current phase of the project.
\end{abstract}

\section{INTRODUCTION}

Fort Rodd Hill National Historic Site in Victoria, British Columbia is currently undergoing a multi-year rehabilitation of its buildings and defensive works. The site was predominantly constructed in the 1890's as part of an elaborate coastal defensive network protecting Esquimalt harbour and naval base. The site was decommissioned in 1956, and designated as a National Historic Site in 1958. (Historic Places, 2009)

The majority of the structures on site consist of un-reinforced massive concrete buildings contained within concrete fortification walls, arranged in three separate coastal batteries. Each consists of a number of common elements including: gun emplacements, underground powder magazines, observation posts, and varying crew shelters and quarters. Other structures between each battery include support and maintenance buildings, the Engineroom for powering the site and searchlights and the Warrant Officer's Quarters. Various modifications were undertaken during the active operational period of the site, most significantly during World War II (Historic Places, 2009), predominantly consisting of additions to existing batteries using reinforced concrete, and most significantly, a $13 \mathrm{~m}$ tall fire control tower that was added at the Belmont Battery.

Various maintenance programs have been undertaken by the custodian agency, Parks Canada throughout the past six decades, however, no significant rehabilitation or structural upgrades had been completed. Heritage Conservation Services (HCS) of Public Services and Procurement Canada (PSPC) took the lead on the rehabilitation program, first conducting detailed condition and structural assessments followed by the design and implementation for each phase of construction, including contract tender documents consisting of drawings and specifications. The second phase of construction marked a significant departure in the way the tender drawing package was produced and displayed, utilizing photogrammetric models, laser scanning to produce orthographic photos to better communicate the as found conditions and scope of work. Traditionally, contract documents issued by the Canadian federal government for construction or rehabilitation projects consist of two dimensional line drawings, sometimes supplemented with record photographs to help communicate the repair methodology or site specific conditions.

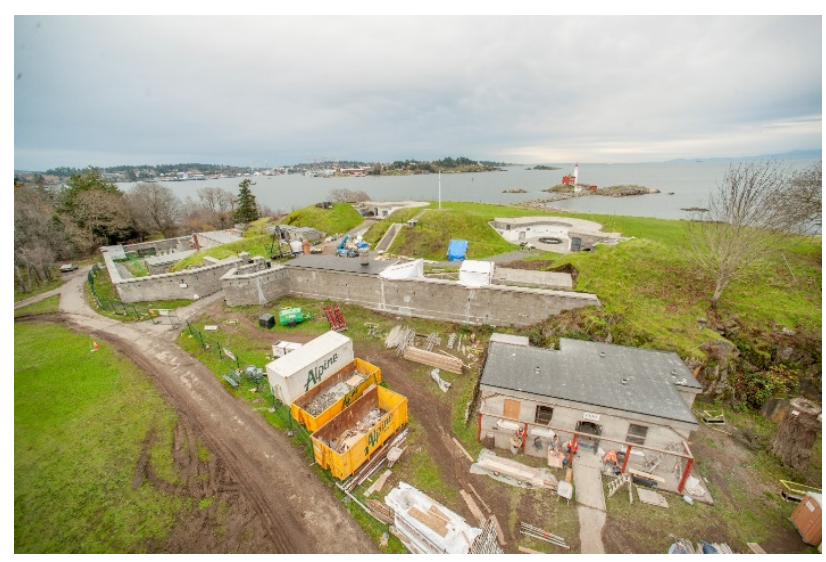

Figure 1: Aerial view of Fort Rodd Hill National Historic Site of Canada Lower Battery rehabilitation.

The latest developments in photogrammetric processing software have resulted in significant efficiencies and cost savings due to the increased clarity and exceptional level of detail contained within the photographic record used to generate the model. The construction site review also included elements of photogrammetric modelling and monitoring to provide ongoing support to the rehabilitation project, adapting to changes to the design, as well as for quality assurance verification.

\section{BODY}

\subsection{Background}

The rehabilitation program of work commenced in the 20092010 fiscal year, beginning with a preliminary condition assessment and heritage documentation project. The resulting drawings consisted of rectified partial elevations of a selection of buildings on site. Of these structures, three exhibiting various levels of deterioration were selected for a pilot project in order to develop effective repair methodologies to maintain as much 
heritage fabric as possible, whilst ensuring the continued viability of the site for decades to come.

The first phase of construction was undertaken on the largest single structure on site, the Casemate Barracks and was tendered with architectural and engineering drawings developed from rectified elevations, 2D line work and record photos. During the corresponding construction and quality review, as well as the investigation and design stages for the next phases, the project team, consisting of the author and Senior HCS Conservation Engineer Doug Stephenson determined an alternate drawing production methodology could benefit the project to more succinctly and completely detail the scope of work.

The drawings for phase two through four of the Fort Rodd Hill rehabilitation project benefitted significantly from the use of the latest Structure-from-Motion (SfM) photogrammetric and laser scanning techniques through the production of highly detailed three dimensional models and the subsequent rendering of orthographic top-plan and elevation images; revealing in quantifiable detail all of the visible deterioration of the exteriors of each structure. The scaled three dimensional models of the structures to be rehabilitated provided information to calculate accurate estimates for repair quantities, correlate interior and exterior deterioration and to ultimately reduce the amount of unknown risk a contractor typically assumes in their tender quotations. The end result of this phase of the project included the efficiency in the production of the contract documents and a significant reduction in capital expenditure and streamlining of the construction due to fewer unexpected conditions.

The heritage documentation work was undertaken in multiple phases during the design process while the author was on-site for quality review visits of the phase one work. A combination of SfM photogrammetry and laser scanning was employed, based on the suitability of the technique for each structure and their propensity to adequately capture the required information (Koska., et al., 2013). Best practices were developed to achieve consistency and maximum efficiency on site to best support the rehabilitation; though this workflow would not necessarily be recommended for detailed documentation projects.

For expediency and efficiency in fulfilling project specific drawing requirements, each building's data was acquired as its own individual model, and not in a site specific coordinate system. The goal was to have a model of each building prior to rehabilitation to serve as a basis for the creation of architectural drawings as well as to serve as posterity record for both quality review purposes and as a mitigation measure for catastrophic loss. As such, the primary concern for each structure was comprehensive photographic coverage and the scalar accuracy of the model.

\subsection{Methodology}

SfM photogrammetry entails multiple photos of a subject captured in a systematic fashion with sufficient overlap between images from numerous locations around the subject, often taken in parallel strips (Westoby., et al., 2012, Niederheiser., et al., 2016). Whereas traditional photogrammetry typically consists of parallel pairs of image frames taken at known distances apart (Chiabrando., et al., 2015). SfM generally relies upon increasingly sophisticated software packages to search for correspondences between the content in photos for 3D reconstruction of the images, also known as photogrammetric or bundle triangulation (To., et al., 2015). Once the image location and orientation has been derived, in relation to the subject, metric information can be readily extracted based on triangulation.

Photographic capture sources can be wide ranging; from terrestrial based cameras such as cell phones, point and shoots, Digital SLRs, infrared cameras or aerial sources such as aircraft, satellite, or Unmanned Aerial Systems (UAS). Each source has their own benefits and limitations, such as cost, optical quality, resolution, noise, speed, image size and coverage. Ultimately, a balance must be struck between each of those factors in order to achieve the desired results.

Like all photogrammetric techniques, SfM relies upon quality photography, meaning sharp focus and adequate exposure. Moreover, it also benefits from today's high resolution cameras with ever improving noise reduction characteristics. Because current camera and lens systems tend to be non-metric, off the shelf products, it can be useful to calibrate each camera, lens and focal range for best results, or to utilize in software calibration estimation parameters to assist in model production. This method also typically requires some form of external scalar control to scale the model appropriately, which can be derived from many sources, including the following examples in decreasing order of precision and accuracy: survey, laser scanning, hand measurements, existing drawings, GPS, etc.

A number of pitfalls can affect the efficiency and final outcome of SfM modeling. With current model full frame 20+ megapixel resolution cameras, file sizes have increased significantly, especially when shooting in RAW formats, therefore, processing requirements and times are greater. Photo sets can be quite substantial in order to achieve the correct photo-scale required to capture sufficient level of detail. Depending on the size of the building, this technique may become less feasible. As well, insufficient or inconsistent ambient lighting can negatively impact the quality or ability of the software to orient the images and reconstruct the model.

The documentation work at Fort Rodd did not require high precision modelling, $20 \mathrm{~mm}$ scalar accuracy was sufficient; however, it was important that the texturized mesh model have a sufficient resolution to display in detail all of the visible deterioration, such as cracks, delamination, erosion, efflorescence, mineral crusts, staining and biological growth. In establishing a standard of requirements, it allowed for efficient and effective site work with known photo coverage parameters in order to achieve the desired results. The photographic field of view required for this project consisted of an image surface coverage of about $2.5 \mathrm{~m}$ by $1.7 \mathrm{~m}$ at $1.5-2 \mathrm{~m}$ backup from the subject using a $20 \mathrm{~mm}$ lens, or approximately 4-5 m backup for a $50 \mathrm{~mm}$ lens. Optimal optical focus throughout the frame is achieved with apertures above f8 and a minimum shutter speed of $1 / 125$ th of a second in ambient light to eliminate camera shake, or 1/60th with supplemental lighting. Ideally, using only fixed focal length lenses, the focusing ring should be locked at the appropriate focal range to produce sharp images within the aperture range at the required backup from the subject. Various photogrammetric software packages operate most reliably with $70 \%$ overlap between image pairs. After the photos are oriented, the point cloud and/or mesh is created and texturized at this point the model can be scaled.

Many HCS projects utilize survey control to both scale and register the model to a specific coordinate system, which can entail significant time expenditure. For the purposes of this project six to eight taped distances provided enough redundancy to provide verifiable results. Generally, long distances are preferable, though with tape measured distances one must be 
mindful of tape sag, as well as requiring a second person to hold the tape on the centre of the targets. Based on the sizes of the buildings on site, targets set $5 \mathrm{~m}$ apart was optimal and by placing them at a pre-determined distance it did not need the rigorous field note control that randomly placed variable scales requires. The interiors of each structure were measured using traditional methods due to time constraints in phase two to allow for section line work to be drawn. As the project evolved and methodologies were validated, laser scanning was utilized for phases three and four, eliminating the need for tape scale distances going forward and providing greater accuracy.

\subsection{Site Specific Considerations}

The buildings at Fort Rodd Hill are particularly well suited to photogrammetric modelling, being predominantly single storey fortification structures with surface texture, allowing full coverage terrestrially without requiring elevated access equipment. Use of telescopic masts or handheld monopods with a timer or remote trigger allowed access to the upper portions of each structure.

Multiple remote flashes were utilized in areas with insufficient ambient lighting to ensure proper exposure, however, this can also affect the software's ability to detect correspondence between photos due to changing lighting conditions and differential shadow projections, as a result, it is best to position the flash to reduce differential shadow casting and to diffuse the light sufficiently enough to reduce hot spots.

The RAW photo sets for each building were post-processed for exposure, white balance, highlight and shadow balancing prior to being converted to JPEG files to be registered in Agisoft Photoscan or Bentley Context Capture. The typical workflow begins with photo alignment and optimization before building a dense point cloud followed by meshing the cloud and texturizing the mesh. Roof and elevation ortho-images were generated within Photoscan and/or CloudCompare software packages. Various elements such as wooden roof canopies or retaining walls were removed to provide differing views of the elevation, as well as profiles of adjacent components, grade or other structures.

Each building was captured with a series of between 100-300 photogrammetric images, depending on size or complexity. Typically, each building required between 20 and 40 minutes to capture the photos, another 20 to 30 minutes to adjust the photos for exposure, shadow/highlight and converting any RAW photos to JPEG. Each building was then processed in photogrammetric software, using batch automation for the alignment, dense cloud, mesh and texturization. The creation of the models and the output of orthographic photo elevations required two to five hours of office time, and another two hours to layout the drawings in AutoCAD. Because of reduced survey or on site measurements required for photogrammetric imaging, the resulting drawings took approximately $30 \%$ less time in total to complete than previous rectified photography workflows. This resulted in significant cost and time savings for the production of the drawings

\subsection{Results and Discussion}

There are numerous benefits to ortho-photo production from a 3D model versus the previously rectified elevations, most notably; greater coverage, more reliable profiles, relative scale of non-planar parallel surfaces, and the elimination of misleading information introduced by perpendicular surfaces which are a product of camera perspective (figure 3 shows the orthographic elevation generated from the model and figure 4 shows the rectified elevation from 2010). The elevations shown in figure 3 are more complete than efficiently possible through rectified photography.

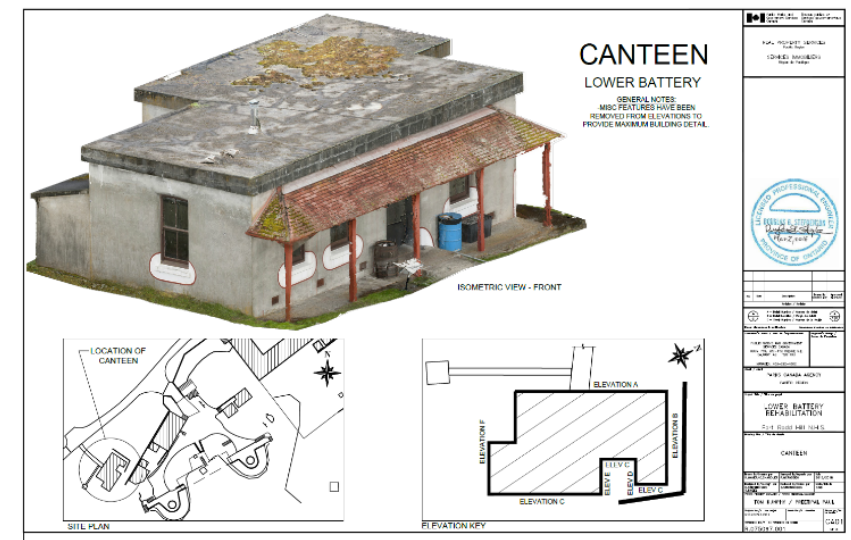

Figure 2: Cover sheet and Isometric image of the Canteen building at Fort Rodd Hill.

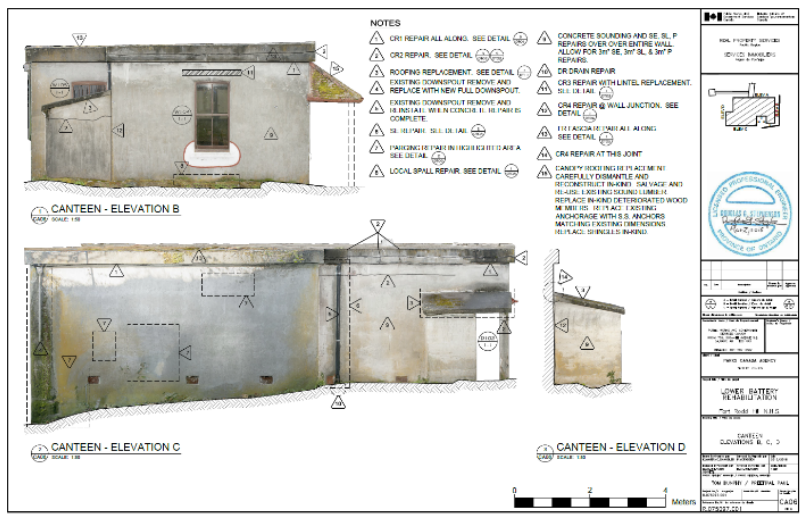

Figure 3: Example of orthographic elevations with designated repairs.

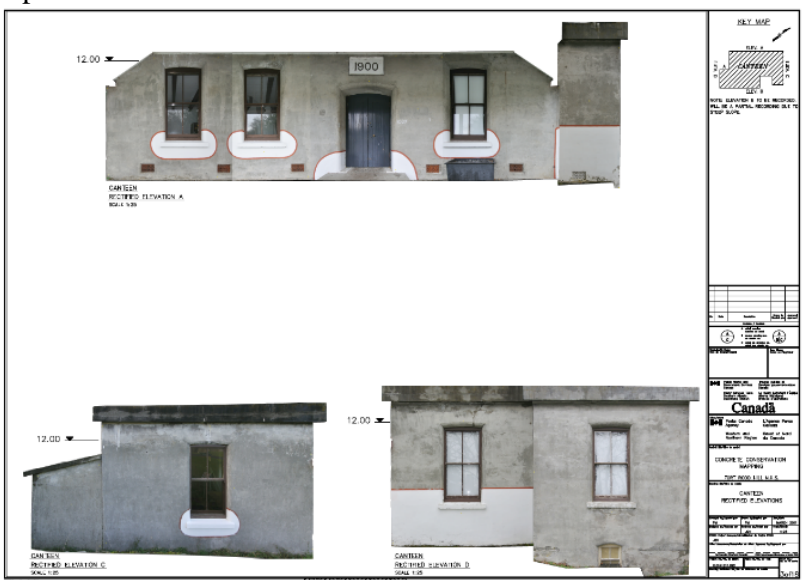

Figure 4: Example of ortho-rectified elevations.

Because the rear elevation of the Canteen building has only a $1 \mathrm{~m}$ gap between a cliff face and the wall, not allowing for sufficient backup to capture the head on photos typically required for rectification. The documentation time on site required for photogrammetry is typically much less than that needed for rectification, which typically requires four measured control points to rectify each photo. Once the model has been generated, each ortho-photo is quickly created and laid out in 
AutoCAD with no further image treatment required. Every visible element or elevation from each structure was laid out to produce a comprehensive record for the transcription of repair notes in a fraction of the time required for photo rectification. The visibility of the exterior deterioration on each elevation made the transcription of repair notes very efficient, complete and ostensibly foolproof. The quantities of each repair type were also calculated more easily to provide more complete information for potential bidders. The new methodology resulted in significant cost savings in the design and tender package production. Based on pre-construction tender estimates for the phase two and three work, the winning bids were significantly less than the estimates because the contractor was able to mitigate the risks typically involved with estimating and bidding on rehabilitation projects.

Further work at the site is to include the use of UAS for aerial photogrammetry and terrestrial laser scanning to document the interior and exterior of all remaining structures and the greater site. This will not only provide the data required to produce contract documents for the final phases of the rehabilitation, it will also provide a posterity record of the entire site.

Each building will be re-photographed after the completion of rehabilitation work in order to produce as-found documentation for maintenance plan and monitoring purposes, with a more rigorous survey and laser scanning methodology, tied to permanent benchmarks to allow for future comparisons using various monitoring techniques.

\section{CONCLUSION}

The example of Fort Rodd Hill can have wide ranging applications in all disciplines of heritage conservation and documentation, not only to provide up front monetary savings, but also a greater breadth of entrained information to assist in design, planning and implementation of a project. This data serves as a detailed posterity record as well as due diligence documentation to help mediate and reduce the occurrences of contractual disputes and resulting settlements.

The value of these emerging digital technologies is becoming well established, resulting in a significant paradigm shift in the workflows of heritage and non-heritage professionals alike. PSPC has readily embraced these data acquisition technologies and is continually exploring emerging methodologies, establishing or refining new workflows as processing solutions evolve.

In June of 2017, the Fort Rodd Hill rehabilitation team was honoured with the PSPC Excellence Award in Innovation and Excellence in Service Delivery for the contribution made to the project resulting in significant cost savings to the Crown in both the design and construction phases of the project. This award is PSPC's highest honour.

\section{REFERENCES}

Chiabrando, F., Donadio, E., Rinaudo, F., 2015. SfM for orthophoto generation: A winning approach for cultural heritage knowledge. In: International Archives of the Photogrammetry, Remote Sensing and Spatial Information Sciences, Taipei, Taiwan, CommissionVI, WG VI/4, pp. 91-98.

Historic Places, 2009. "Fort Rodd Hill National Historic Site of Canada," Ottawa, Ontario http://www.historicplaces.ca/en/repreg/place-lieu.aspx?id=13469\&pid=0
Koska, B., Kremen, T., 2013. The combination of laser scanning and structure from motion technology for creation of accurate exterior and interior orthophotos of St. Nicholas baroque church. In: International Archives of the Photogrammetry, Remote Sensing and Spatial Information Sciences, Trento, Italy, Vol. XL-5/W1, pp. 133-138.

Niederheiser, R., Mokros, M., Lange, J., Petschko, H., Prasicek, G., Oude Elberink, S., 2016. Deriving 3D point clouds from terrestrial photographs- comparison of different sensors and software. In: International Archives of the Photogrammetry, Remote Sensing and Spatial Information Sciences, Berlin, Germany, Vol. XLI-B5, pp. 685-692.

To, T., Nguyen, D., Tran, G., 2015. Automated 3D architecture reconstruction from photogrammetric structure and motion: A case study of the "One Pilla” Pagoda, Hanoi, Vietnam. In: International Archives of the Photogrammetry, Remote Sensing and Spatial Information Sciences, Berlin, Germany, Vol. XL7/W3, pp. 1425-1429.

Westoby, M.J., Brasington, J., Glasser, N.F., Hambrey, M.J., Reynolds, J.M., 2012. "Structure-from-Motion" photogrammetry: A low-cost, effective tool for geoscience applications. Geomorphology 179, 300-314. 